\title{
Evaluation of Various Enzymes Supplementation on Nutrient Utilization and Efficiency Parameters on Rice Gluten Meal Based Diets in Broilers
}

\author{
Om Prakash Dinani*, Pramod Kumar Tyagi, Chandra Deo, S.K. Bhanja and J.J. Rokade \\ Avian Nutrition and Feed Technology Division, ICAR-Central Avian Research Institute, U.P., INDIA \\ *Corresponding author: OP Dinani; E-mail:dr_dinani@rediffmail.com
}

Received: 16 June, 2020

Revised: 23 July, 2020

Accepted: 29 July, 2020

\begin{abstract}
A biological experiment of 42 days duration was undertaken in day old chicks $(n=384)$ divided into 12 dietary treatments as per $3 \times 4$ factorial design having 4 replicates per treatment with 8 birds in each. Twelve experimental diets were prepared by incorporating control, two different levels of rice gluten meal (RGM) consisted of (15 and 17.5\%), without and with three different types of enzymes xylanase, protease and multienzymes. The nutrient utilization in terms of nitrogen retention, apparent metabolizable energy of the diets, dry matter and gross energy metabolizability were significantly $(\mathrm{P}<0.01)$ lower at $17.5 \%$ level as compared to 0 and $15 \%$ RGM levels. The dry matter metabolizability and nitrogen retention of the birds were significantly $(\mathrm{P}<0.01)$ better in protease enzyme supplemented groups compared to control and other enzyme supplemented groups. The interaction of RGM and enzymes showed no significant $(\mathrm{P}>0.05)$ difference in nutrient utilization between different dietary treatments and control in gross energy metabolizability, calcium and phosphorous retention. The protein and energy efficiency ratios of birds were not influenced by RGM feeding, whereas significant $(\mathrm{P}<0.05)$ improvement were observed in protease supplemented birds. Thus, it may be concluded that protease enzyme supplementation was found best in RGM diet to improve their feeding value and it may be used to increase the effective and safe inclusion level from 15 to $17.5 \%$ in broiler diets.
\end{abstract}

\section{HIGHLIGHTS}

(0 Rice gluten meal may be used up to $15 \%$ level without affecting $(\mathrm{P}>0.05)$ nutrient utilization.

(- Protein and energy efficiencies improvement was significantly observed in protease supplemented birds.

Keywords: Enzymes, Efficiency parameters, Nutrient utilization

The search of alternative feed ingredients in poultry nutrition is a continuous process in the pursuit of economical poultry production. India is the second largest producers of rice in the world after China, producing approximately 109.7 MT rice in 2016-17 (Agriculture Statistics, 2018). Now-a-days, certain newer rice by products are available in appreciable quantities from rice processing industries and at cheaper rate such as rice gluten meal (RGM), which can be utilized in feeding poultry. The RGM is a by-product of wet-milling of rice obtained after starch extraction and syrup preparation. It is relatively a newer feedstuff having brownish color and coarse powdery texture. Commercial traders categorise RGM as a high crude protein and energy ingredient which is priced lower than soybean meal (Wani, 2018).
Strategic development of substrate specific suitable enzyme in diet will enhance the nutritive value of diets (Chesson, 2001). No information is available on the appropriate enzyme that is specific for broiler diets based soybean meal partially replaced with RGM.

Initial research finding showed that RGM can be included up to $10 \%$ level in broiler chicken without affecting feed efficiency and dressing percentage (Sherazi et al., 1995). Metwally and Farahat (2015) found that broiler fed RGM

How to cite this article: Dinani, O.P., Tyagi, P.K., Deo, C., Bhanja, S.K. and Rokade, J.J. (2020). Evaluation of various enzymes supplementation on nutrient utilization and efficiency parameters on rice gluten meal based diets in broilers. J. Anim. Res., 10(4): 515-523.

Source of Support: None; Conflict of Interest: None 
with different inclusion rates up to $12.5 \%$ had the same growth performance. Kumar et al. (2016) found that RGM could replace groundnut cake in the concentrate mixture of growing calves up to $75 \%$ level without any adverse effects on growth performance and nutrient utilization. Malik et al. (2017) reported that replacement of groundnut cake by RGM and maize gluten meal at $75 \%$ level did not affect the DM intake, feed efficiency and average daily gain in growing Sahiwal cattle.

Overall, very little research data is available on feeding value of RGM in poultry and no data is available regarding evaluation of various enzymes supplementation on nutrient utilization and efficiency parameters on rice gluten meal based diets in broilers.

Thus, this study was conducted for substract specific selection of enzyme for RGM and its effect on the nutrient utilization and efficiency parameters of broiler chicken production.

\section{MATERIALS AND METHODS}

\section{Ethical approval}

The research work was carried out at the Division of Avian Nutrition and Feed Technology, ICAR-Central Avian Research Institute (CARI), Izatnagar, India as per the guidelines and approval of Institute Animal Ethical Committee (IAEC) and Committee for the Purpose of
Control and Supervision of Experiments on Animals (CPCSEA).

\section{Experimental design}

Experimental layout for feeding different level of RGM with or without enzymes is presented in Table 1 . The experiment was conducted as per $3 \times 4$ factorial completely randomized design (CRD) in 384 broiler chicks (CARIBRO vishal). The birds were randomly divided into 48 replicates of eight birds each. There were twelve different treatments with 4 replicates for each treatment. Two levels of rice gluten meal were taken, the best inclusion level from earlier experiments as first level $(15 \%)$ and then adding over and above the best level of $2.5 \%$ RGM to this level. Protease, xylanase and multienzymes supplementation under different treatments were used to find out the most suitable enzyme for RGM diets.

\section{Experimental diets}

Analyzed chemical composition of dietary ingredients (\%) as per (AOAC, 2000) on as such basis is presented in Table 2 . Ingredients and nutrient composition (\%) of pre-starter (0-14 days), starter (14-28 days) and finisher (28-42 days) diets with or without enzymes for different level of RGM are presented respectively in Table 3, 4 and 5 as per ICAR (2013) standard. In vitro pepsin-pancreatin digestibilities of RGM and soybean meal were measured according to

Table 1: Experimental layout for feeding different level of RGM with or without enzymes

\begin{tabular}{llllll}
\hline & \multicolumn{2}{c}{ Experimental design } & \multicolumn{2}{c}{$\mathbf{3 \times 4}$ factorial CRD } \\
\hline Treatment & Rice gluten meal (\%) & No. of replicates & Birds/ replication & Total & Enzymes \\
\hline T1 & 0.0 & 4 & 8 & 32 & - \\
T2 & 0.0 & 4 & 8 & 32 & Xylanase \\
T3 & 0.0 & 4 & 8 & 32 & Protease \\
T4 & 0.0 & 4 & 8 & 32 & Multienzymes \\
T5 & 15 & 4 & 8 & 32 & - \\
T6 & 15 & 4 & 8 & 32 & Xylanase \\
T7 & 15 & 4 & 8 & 32 & Protease \\
T8 & 15 & 4 & 8 & 32 & Multienzymes \\
T9 & 17.5 & 4 & 8 & 32 & - \\
T10 & 17.5 & 4 & 8 & 32 & Xylanase \\
T11 & 17.5 & 4 & 8 & 32 & Protease \\
T12 & 17.5 & 4 & 8 & 32 & Multienzymes \\
\hline
\end{tabular}


Table 2: Analyzed chemical composition of dietary ingredients (\%) on as such basis

\begin{tabular}{|c|c|c|c|c|c|c|c|c|c|c|c|}
\hline Ingredients & Moisture & DM & $\mathbf{C P}$ & EE & $\mathbf{C F}$ & TA & NFE & $\mathbf{C a}$ & $\mathbf{P}$ & GE (kcal/kg) & *ME (kcal/kg) \\
\hline Maize & 8.6 & 91.3 & 9 & 3.9 & 1.8 & 1.4 & 83.8 & 0.03 & 0.29 & 4447 & 3350 \\
\hline SBM & 9.1 & 90.9 & 44.5 & 0.9 & 6.2 & 3.1 & 45.2 & 0.32 & 0.68 & 4097 & 2400 \\
\hline DORB & 10.1 & 91.8 & 14 & 1.6 & 15.9 & 5.8 & 62.6 & 0.3 & 1.54 & 3854 & 2000 \\
\hline RGM & 7.6 & 92.3 & 49.9 & 5.7 & 7.4 & 3.3 & 33.5 & 0.84 & 0.98 & 4742 & 3031 \\
\hline Soybean oil & - & - & - & - & - & - & - & - & - & 8900 & 8450 \\
\hline Lime stone powder & 1.4 & 98.6 & - & - & - & - & - & 33.89 & - & - & - \\
\hline Marbal chip & 1.3 & 98.7 & - & - & - & - & - & 33.84 & - & - & - \\
\hline DCP & 7.2 & 92.7 & - & - & - & - & - & 22.92 & 16.04 & - & - \\
\hline
\end{tabular}

*Calculated value.

Table 3: Ingredients and nutrient composition (\%) of pre-starter diets with or without enzymes for different level of RGM

\begin{tabular}{|c|c|c|c|c|c|c|c|c|c|c|c|c|}
\hline Ingredients & T1 & T2 & T3 & T4 & T5 & T6 & T7 & T8 & T9 & T10 & T11 & T12 \\
\hline Maize & 54.42 & 54.42 & 54.42 & 54.42 & 59.40 & 59.40 & 59.40 & 59.40 & 60.00 & 60.00 & 60.00 & 60.00 \\
\hline SBM & 38.40 & 38.40 & 38.40 & 38.40 & 20.70 & 20.70 & 20.70 & 20.70 & 17.80 & 17.80 & 17.80 & 17.80 \\
\hline RGM & 0.00 & 0.00 & 0.00 & 0.00 & 15.00 & 15.00 & 15.00 & 15.00 & 17.50 & 17.50 & 17.50 & 17.50 \\
\hline Oil & 3.00 & 3.00 & 3.00 & 3.00 & 0.70 & 0.70 & 0.70 & 0.70 & 0.40 & 0.40 & 0.40 & 0.40 \\
\hline LSP & 1.40 & 1.40 & 1.40 & 1.40 & 1.30 & 1.30 & 1.30 & 1.30 & 1.30 & 1.30 & 1.30 & 1.30 \\
\hline DCP & 1.82 & 1.82 & 1.82 & 1.82 & 1.95 & 1.95 & 1.95 & 1.95 & 1.95 & 1.95 & 1.95 & 1.95 \\
\hline Lysine & 0.00 & 0.00 & 0.00 & 0.00 & 0.12 & 0.12 & 0.12 & 0.12 & 0.18 & 0.18 & 0.18 & 0.18 \\
\hline Methionine & 0.20 & 0.20 & 0.20 & 0.20 & 0.06 & 0.06 & 0.06 & 0.06 & 0.06 & 0.06 & 0.06 & 0.06 \\
\hline Constant* & 0.765 & 0.765 & 0.765 & 0.765 & 0.765 & 0.765 & 0.765 & 0.765 & 0.765 & 0.765 & 0.765 & 0.765 \\
\hline Enzyme & - & + & + & + & - & + & + & + & - & + & + & + \\
\hline Total & 100.01 & 100.01 & 100.01 & 100.01 & 100.00 & 100.00 & 100.00 & 100.00 & 100.00 & 100.00 & 100.00 & 100.00 \\
\hline \multicolumn{13}{|c|}{ Nutrient composition } \\
\hline$\overline{\mathrm{CP}}$ & 21.99 & 21.99 & 21.99 & 21.99 & 22.06 & 22.06 & 22.06 & 22.06 & 22.07 & 22.07 & 22.07 & 22.07 \\
\hline Lysine & 1.19 & 1.19 & 1.19 & 1.19 & 1.20 & 1.20 & 1.20 & 1.20 & 1.21 & 1.21 & 1.21 & 1.21 \\
\hline Methionine & 0.52 & 0.52 & 0.52 & 0.52 & 0.52 & 0.52 & 0.52 & 0.52 & 0.54 & 0.54 & 0.54 & 0.54 \\
\hline Threonine & 0.81 & 0.81 & 0.81 & 0.81 & 0.83 & 0.83 & 0.83 & 0.83 & 0.82 & 0.82 & 0.82 & 0.82 \\
\hline $\mathrm{Ca}$ & 1.03 & 1.03 & 1.03 & 1.03 & 1.01 & 1.01 & 1.01 & 1.01 & 1.01 & 1.01 & 1.01 & 1.01 \\
\hline $\mathrm{P}$ & 0.45 & 0.45 & 0.45 & 0.45 & 0.45 & 0.45 & 0.45 & 0.45 & 0.44 & 0.44 & 0.44 & 0.44 \\
\hline $\operatorname{ME}(\mathrm{kcal} / \mathrm{kg})^{* *}$ & 2998 & 2998 & 2998 & 2998 & 3001 & 3001 & 3001 & 3001 & 3001 & 3001 & 3001 & 3001 \\
\hline Cost (₹/kg) & 28.52 & 29.03 & 29.13 & 28.93 & 24.68 & 25.19 & 25.29 & 25.09 & 24.31 & 24.82 & 24.92 & 24.72 \\
\hline
\end{tabular}

In prestarter diet $*$ Constant 0.765 includes salt $0.4 \%$, trace mineral premix $0.1 \%$, vitamin premix $0.15 \%$, vit. B complex $0.015 \%$, choline chloride $0.05 \%$ and Toxin binder $0.05 \%$. Trace mineral premix supplied $\mathrm{mg} / \mathrm{kg}$ diet: $\mathrm{Mn}, 55 ; \mathrm{I}, 1 ; \mathrm{Fe}, 75 ; \mathrm{Zn}, 60 ; \mathrm{Cu}, 10 ; \mathrm{Se}, 0.15 \mathrm{and} \mathrm{Cr}$, 0.2 . The vitamin premix supplied per kg diet: Vit.A, 5000 IU; Vit.D 3 , 2400 IU; Vit.E, 15 and Vit. K, 1mg. Vitamin B complex supplied per kg diet: Vit. $\mathrm{B}_{1}, 5 \mathrm{mg}$; Vit. $\mathrm{B}_{2}, 6 \mathrm{mg}$; Vit. $\mathrm{B}_{6} 5 \mathrm{mg}$; Vit. $\mathrm{B}_{12}, 15 \mathrm{mcg}$; nicotinic acid, $35 \mathrm{mg}$; pantothenic acid, $12 \mathrm{mg}$; biotin $0.15 \mathrm{mg}$ and folic acid $0.5 \mathrm{mg}$. Choline chloride supplied per kg diet: choline, $1300 \mathrm{mg}$. (As per ICAR, 2013) **calculated value.

Table 4: Ingredients and nutrient composition (\%) of starter diets with or without enzymes for different level of RGM

\begin{tabular}{|c|c|c|c|c|c|c|c|c|c|c|c|c|}
\hline Ingredients & T1 & T2 & T3 & T4 & T5 & T6 & T7 & T8 & T9 & T10 & T11 & T12 \\
\hline Maize & 55.63 & 55.63 & 55.63 & 55.63 & 60.70 & 60.70 & 60.70 & 60.70 & 61.62 & 61.62 & 61.62 & 61.62 \\
\hline SBM & 37.10 & 37.10 & 37.10 & 37.10 & 19.20 & 19.20 & 19.20 & 19.20 & 16.20 & 16.20 & 16.20 & 16.20 \\
\hline RGM & 0.00 & 0.00 & 0.00 & 0.00 & 15.00 & 15.00 & 15.00 & 15.00 & 17.50 & 17.50 & 17.50 & 17.50 \\
\hline oil & 3.50 & 3.50 & 3.50 & 3.50 & 1.20 & 1.20 & 1.20 & 1.20 & 0.80 & 0.80 & 0.80 & 0.80 \\
\hline
\end{tabular}




\begin{tabular}{|c|c|c|c|c|c|c|c|c|c|c|c|c|}
\hline LSP & 1.35 & 1.35 & 1.35 & 1.35 & 1.32 & 1.32 & 1.32 & 1.32 & 1.32 & 1.32 & 1.32 & 1.32 \\
\hline DCP & 1.55 & 1.55 & 1.55 & 1.55 & 1.70 & 1.70 & 1.70 & 1.70 & 1.70 & 1.70 & 1.70 & 1.70 \\
\hline Lysine & 0.00 & 0.00 & 0.00 & 0.00 & 0.06 & 0.06 & 0.06 & 0.06 & 0.06 & 0.06 & 0.06 & 0.06 \\
\hline Methionine & 0.10 & 0.10 & 0.10 & 0.10 & 0.03 & 0.03 & 0.03 & 0.03 & 0.03 & 0.03 & 0.03 & 0.03 \\
\hline Constant* & 0.765 & 0.765 & 0.765 & 0.765 & 0.765 & 0.765 & 0.765 & 0.765 & 0.765 & 0.765 & 0.765 & 0.765 \\
\hline Enzyme & - & + & + & + & - & + & + & + & - & + & + & + \\
\hline Total & 100.00 & 100.00 & 100.00 & 100.00 & 100.0 & 100.0 & 100.0 & 100.0 & 100.00 & 100.00 & 100.00 & 100.00 \\
\hline \multicolumn{13}{|c|}{ Nutrient composition } \\
\hline $\mathrm{CP}$ & 21.52 & 21.52 & 21.52 & 21.52 & 21.51 & 21.51 & 21.51 & 21.51 & 21.50 & 21.50 & 21.50 & 21.50 \\
\hline Lysine & 1.38 & 1.38 & 1.38 & 1.38 & 1.09 & 1.09 & 1.09 & 1.09 & 1.04 & 1.04 & 1.04 & 1.04 \\
\hline Methionine & 0.48 & 0.48 & 0.48 & 0.48 & 0.49 & 0.49 & 0.49 & 0.49 & 0.50 & 0.50 & 0.50 & 0.50 \\
\hline Threonine & 0.80 & 0.80 & 0.80 & 0.80 & 0.79 & 0.79 & 0.79 & 0.79 & 0.81 & 0.81 & 0.81 & 0.81 \\
\hline $\mathrm{Ca}$ & 0.95 & 0.95 & 0.95 & 0.95 & 0.95 & 0.95 & 0.95 & 0.95 & 0.95 & 0.95 & 0.95 & 0.95 \\
\hline $\mathrm{P}$ & 0.41 & 0.41 & 0.41 & 0.41 & 0.40 & 0.40 & 0.40 & 0.40 & 0.40 & 0.40 & 0.40 & 0.40 \\
\hline $\operatorname{ME}(\mathrm{kcal} / \mathrm{kg})^{* *}$ & 3050 & 3050 & 3050 & 3050 & 3050 & 3050 & 3050 & 3050 & 3051 & 3051 & 3051 & 3051 \\
\hline Cost (₹/ kg) & 28.03 & 28.53 & 28.63 & 28.43 & 24.43 & 25.03 & 24.93 & 24.83 & 23.86 & 24.46 & 24.36 & 24.26 \\
\hline
\end{tabular}

In starter diet $*$ Constant 0.765 includes salt $0.4 \%$, trace mineral premix $0.1 \%$, vitamin premix $0.15 \%$, vit. B complex $0.015 \%$, choline chloride $0.05 \%$ and Toxin binder $0.05 \%$. Trace mineral premix supplied mg / kg diet: $\mathrm{Mn}, 55 ; \mathrm{I}, 1 ; \mathrm{Fe}, 60 ; \mathrm{Zn}, 60 ; \mathrm{Cu}, 10 ; \mathrm{Se}, 0.15 \mathrm{and} \mathrm{Cr}$, 0.2. The vitamin premix supplied per kg diet: Vit.A, $5000 \mathrm{IU}$; Vit.D 3 , $2400 \mathrm{IU}$; Vit.E, 15 and Vit.K, $1 \mathrm{mg}$. Vitamin B complex supplied per kg diet: Vit. $\mathrm{B}_{1}, 4 \mathrm{mg}$; Vit. $\mathrm{B}_{2}, 6 \mathrm{mg}$; Vit. $\mathrm{B}_{6} 5 \mathrm{mg}$; Vit. $\mathrm{B}_{12}, 15 \mathrm{mcg}$; nicotinic acid, $35 \mathrm{mg}$; pantothenic acid, $10 \mathrm{mg}$; biotin $0.15 \mathrm{mg}$ and folic acid $0.5 \mathrm{mg}$. Choline chloride supplied per kg diet: choline, $1200 \mathrm{mg}$. (As per ICAR, 2013) **calculated value.

Table 5: Ingredients and nutrient composition (\%) of finisher diets with or without enzymes for different level of RGM

\begin{tabular}{|c|c|c|c|c|c|c|c|c|c|c|c|c|}
\hline Ingredients & $\mathrm{T} 1$ & $\mathrm{~T} 2$ & T3 & T4 & T5 & T6 & $\mathrm{T7}$ & $\mathrm{T} 8$ & T9 & T10 & T11 & T12 \\
\hline Maize & 62.00 & 62.00 & 62.00 & 62.00 & 67.07 & 67.07 & 67.07 & 67.07 & 67.97 & 67.97 & 67.97 & 67.97 \\
\hline SBM & 31.30 & 31.30 & 31.30 & 31.30 & 13.40 & 13.40 & 13.40 & 13.40 & 10.40 & 10.40 & 10.40 & 10.40 \\
\hline RGM & 0.00 & 0.00 & 0.00 & 0.00 & 15.00 & 15.00 & 15.00 & 15.00 & 17.50 & 17.50 & 17.50 & 17.50 \\
\hline oil & 3.22 & 3.22 & 3.22 & 3.22 & 0.90 & 0.90 & 0.90 & 0.90 & 0.50 & 0.50 & 0.50 & 0.50 \\
\hline LSP & 0.00 & 0.00 & 0.00 & 0.00 & 0.00 & 0.00 & 0.00 & 0.00 & 0.00 & 0.00 & 0.00 & 0.00 \\
\hline $\mathrm{DCP}$ & 1.45 & 1.45 & 1.45 & 1.45 & 1.60 & 1.60 & 1.60 & 1.60 & 1.60 & 1.60 & 1.60 & 1.60 \\
\hline Lysine & 0.00 & 0.00 & 0.00 & 0.00 & 0.12 & 0.12 & 0.12 & 0.12 & 0.12 & 0.12 & 0.12 & 0.12 \\
\hline Methionine & 0.06 & 0.06 & 0.06 & 0.06 & 0.00 & 0.00 & 0.00 & 0.00 & 0.00 & 0.00 & 0.00 & 0.00 \\
\hline Marbal Chips & 1.20 & 1.20 & 1.20 & 1.20 & 1.14 & 1.14 & 1.14 & 1.14 & 1.14 & 1.14 & 1.14 & 1.14 \\
\hline Constant* & 0.765 & 0.765 & 0.765 & 0.765 & 0.765 & 0.765 & 0.765 & 0.765 & 0.765 & 0.765 & 0.765 & 0.765 \\
\hline Total & 100.00 & 100.00 & 100.00 & 100.00 & 100.00 & 100.00 & 100.00 & 100.00 & 100.00 & 100.00 & 100.00 & 100.00 \\
\hline \multicolumn{13}{|c|}{ Nutrient composition } \\
\hline $\mathrm{CP}$ & 19.51 & 19.51 & 19.51 & 19.51 & 19.50 & 19.50 & 19.50 & 19.50 & 19.50 & 19.50 & 19.50 & 19.50 \\
\hline Lysine & 1.20 & 1.20 & 1.20 & 1.20 & 0.98 & 0.98 & 0.98 & 0.98 & 0.92 & 0.92 & 0.92 & 0.92 \\
\hline Methionine & 0.41 & 0.41 & 0.41 & 0.41 & 0.43 & 0.43 & 0.43 & 0.43 & 0.44 & 0.44 & 0.44 & 0.44 \\
\hline Threonine & 0.86 & 0.86 & 0.86 & 0.86 & 0.81 & 0.81 & 0.81 & 0.81 & 0.80 & 0.80 & 0.80 & 0.80 \\
\hline $\mathrm{Ca}$ & 0.86 & 0.86 & 0.86 & 0.86 & 0.85 & 0.85 & 0.85 & 0.85 & 0.85 & 0.85 & 0.85 & 0.85 \\
\hline $\mathrm{P}$ & 0.38 & 0.38 & 0.38 & 0.38 & 0.38 & 0.38 & 0.38 & 0.38 & 0.37 & 0.37 & 0.37 & 0.37 \\
\hline ME** & 3100.3 & 3100.3 & 3100.3 & 3100.3 & 3099.1 & 3099.1 & 3099.1 & 3099.1 & 3099.3 & 3099.3 & 3099.3 & 3099.3 \\
\hline Cost (₹/kg) & 2672 & 2672 & 2672 & 2672 & 2584 & 2584 & 2584 & 2584 & 2526 & 2526 & 2526 & 2526 \\
\hline
\end{tabular}

In finisher diet $*$ Constant 0.77 includes salt $0.4 \%$, trace mineral premix $0.1 \%$, vitamin premix $0.15 \%$, vit. B complex $0.015 \%$, choline chloride $0.05 \%$ and Toxin binder $0.05 \%$. Trace mineral premix supplied mg / kg diet: Mn, 50; I, 1; Fe, 50; Zn, 60; Cu, 8; Se, 0.15 and Cr,0.2. The vitamin premix supplied per kg diet: Vit.A, 5000 IU; Vit.D 3 , 2400 IU; Vit.E, 15 and Vit. K, 0.8 mg. Vitamin B complex supplied per kg diet: Vit. $\mathrm{B}_{1}, 4 \mathrm{mg}$; Vit. $\mathrm{B}_{2}, 6 \mathrm{mg}$; Vit. $\mathrm{B}_{6} 5 \mathrm{mg}$; Vit.B $12,15 \mathrm{mcg}$; nicotinic acid, $30 \mathrm{mg}$; pantothenic acid, $10 \mathrm{mg}$; biotin $0.15 \mathrm{mg}$ and folic acid $0.5 \mathrm{mg}$. Choline chloride supplied per kg diet: choline, $900 \mathrm{mg}$. (As per ICAR, 2013) **alculated value. 
the method of Gopalkrishnan and Jamuna (2000). IVPPD of RGM was found $81.92 \%$ and that of soybean meal was $88.15 \%$. No detectable aflatoxin B1 and ochratoxin were found in RGM as estimated by thin layer chromatography (AOAC, 2000).

The three commercial enzyme preparations protease, xylanase and multienzymes were used as per manufacturer instructions after assessing their activity as per standard methods complied by Kamra and Agarwal (2003). Protease activity was estimated $600,000 \pm 849$ units per g. Xylanase activity was estimated $150,000 \pm 683$ units per g. Multienzymes activity were estimated cellulase 15,000 , xylanase $18,500 \pm 328$, beta glucanase $12,500 \pm 128$, amylase $1500 \pm 46$, pectinase $150 \pm 16$, protease $5000 \pm 136$, lipase $15 \pm 3.8$ and beta mannanase $400 \pm 31$. Mixing ratio $50 \mathrm{~g}$ per $100 \mathrm{~kg}$ feed for protease, $10 \mathrm{~g}$ per $100 \mathrm{~kg}$ feed for xylanase and $25 \mathrm{~g}$ per $100 \mathrm{~kg}$ feed for multienzymes were used as per manufacturer instructions.

\section{Metabolism trial}

A four day metabolism trial was conducted to study the utilization of dry matter, nitrogen, gross energy metabolizability (GEM), calcium, phosphorous, and apparent metabolizable energy (AME) from $24^{\text {th }}$ to $27^{\text {th }}$ day of the feeding trial in all the birds. In order to study the utilization of nutrients, a metabolism trial of four days was conducted at $4^{\text {th }}$ week of age $\left(24^{\text {th }}\right.$ to $27^{\text {th }}$ day) during which the net feed consumed by each group of birds in the respective dietary treatment was recorded and the dropping voided over same period were collected quantitatively. The representative sample of test diets and excreta samples were ground and stored in air tight containers for further analysis. The intake, excreted and retained amount of nutrient was calculated $\mathrm{g} / \mathrm{b} / \mathrm{d}$ basis and $\%$ retention of nutrient was calculated on the basis of total intake. The retention of nutrients was calculated by following formula:

Nutrient retention $(\%)=$ Nutrient intake-nutrient outgo/ Nutrient intake $\times 100$

Protein Efficiency Ratio (PER): Weight gain (g)/Protein intake $(\mathrm{g}) \times 100$

Energy Efficiency Ratio (EER): Weight gain (g)/Total ME intake $(\mathrm{kcal}) \times 100$

\section{Statistical Analysis}

Data subjected to test of significance as per $3 \times 4$ factorial completely randomized design (CRD) were analyzed for mean, standard errors and analysis of variance by Snedecor and Cochran (1989) using statistical package for social sciences (SPSS) 16.0 version and comparison of means were done using Tukey's test (1949).

\section{RESULTS AND DISCUSSION}

The results pertaining to nutrient utilization as affected by feeding different levels of RGM with or without enzymes are presented in Table 6 . The results revealed that no significant $(\mathrm{P}>0.05)$ difference was found in calcium and phosphorous retention (\%) by incorporating different levels of RGM at 0,15 and $17.5 \%$ levels. Dry matter metabolizability (DMM), nitrogen retention and apparent metabolizable energy (AME) of the diets were significantly $(\mathrm{P}<0.01)$ decreased in $17.5 \%$ level as compared to 0 and 15\% RGM levels. Gross energy metabolizability (GEM) was significantly $(\mathrm{P}<0.05)$ decreased in $17.5 \%$ level as compared to 0 and $15 \%$ RGM levels.

Effect of with or without enzymes on nutrient utilization revealed that no significant $(\mathrm{P}>0.05)$ difference was observed between treatments in calcium and phosphorous retention (\%) in enzymes (X, P and $\mathrm{M}$ ) supplemented groups as compared to without enzyme supplemented groups. Dry matter metabolizability (DMM) was significantly $(\mathrm{P}<0.01)$ better in xylanase and protease enzyme groups as compared to without enzyme and multienzymes groups. Protease enzyme supplemented groups were significantly $(\mathrm{P}<0.01)$ better as compared to without enzyme and with enzymes (X, P and $\mathrm{M}$ ) supplemented groups in terms of dry matter metabolizability. Nitrogen retention (\%) was significantly $(\mathrm{P}<0.05)$ better in protease enzyme supplemented groups as compared to without and with enzymes (X and $\mathrm{M}$ ) supplemented groups. Gross energy metabolizability (GEM) was significantly $(\mathrm{P}<0.01)$ better in xylanase enzyme supplemented group as compared to without enzyme, protease and multienzymes supplemented groups. However, protease enzyme supplementation was significantly $(\mathrm{P}<0.01)$ better than without and multienzymes supplemented groups in terms of GEM (\%). Apparent metabolizable energy (AME) of the diets were significantly $(\mathrm{P}<0.01)$ better in xylanase 
Table 6: Effect of feeding different level of RGM with or without enzymes on nutrient utilization (\%)

\begin{tabular}{|c|c|c|c|c|c|c|c|c|}
\hline Treatment & RGM \% & Enzyme & DM & Nitrogen & GEM & $\mathbf{C a}$ & $\mathbf{P}$ & AME(kcal/kg) \\
\hline $\mathrm{T} 1$ & 0 & - & $72.5^{b}$ & $55.9^{\mathrm{bc}}$ & 76.1 & 34.5 & 37.9 & $3168^{a}$ \\
\hline $\mathrm{T} 2$ & 0 & $\mathrm{X}$ & $75.6^{\mathrm{d}}$ & $56.02^{\mathrm{bc}}$ & 78.6 & 34.6 & 39.8 & $3231^{\mathrm{b}}$ \\
\hline $\mathrm{T} 3$ & 0 & $\mathrm{P}$ & $76.1^{\mathrm{d}}$ & $56.7^{\mathrm{bc}}$ & 78.2 & 32.8 & 41.0 & $3163^{\mathrm{a}}$ \\
\hline $\mathrm{T} 4$ & 0 & M & $72.2^{b}$ & $57.1^{\mathrm{c}}$ & 75.7 & 32.6 & 38.5 & $3257^{\mathrm{b}}$ \\
\hline T5 & 15 & - & $72.5^{b}$ & $56.6^{\mathrm{c}}$ & 76.6 & 32.2 & 40.5 & $3168^{a}$ \\
\hline T6 & 15 & $\mathrm{X}$ & $75.6^{d}$ & $56.9^{c}$ & 78.2 & 34.4 & 40.5 & $3231^{\mathrm{b}}$ \\
\hline $\mathrm{T} 7$ & 15 & $\mathrm{P}$ & $76.2^{\mathrm{d}}$ & $57.7^{\mathrm{c}}$ & 77.2 & 34.5 & 38.6 & $3257^{\mathrm{b}}$ \\
\hline $\mathrm{T} 8$ & 15 & M & $72.2^{b}$ & $55.9^{\mathrm{bc}}$ & 76.6 & 34.3 & 39.4 & $3164^{a}$ \\
\hline T9 & 17.5 & - & $71.0^{\mathrm{ab}}$ & $54.1^{\mathrm{a}}$ & 76.0 & 32.6 & 37.9 & $3150^{\mathrm{a}}$ \\
\hline $\mathrm{T} 10$ & 17.5 & $\mathrm{X}$ & $70.2^{\mathrm{a}}$ & $56.6^{\mathrm{c}}$ & 77.7 & 32.2 & 39.8 & $3252^{b}$ \\
\hline $\mathrm{T} 11$ & 17.5 & $\mathrm{P}$ & $74.0^{\mathrm{c}}$ & $57.1^{\mathrm{c}}$ & 75.8 & 34.4 & 38.6 & $3127^{a}$ \\
\hline \multirow[t]{15}{*}{$\mathrm{T} 12$} & 17.5 & M & $70.5^{\mathrm{a}}$ & $55.0^{\mathrm{bc}}$ & 75.7 & 34.6 & 40.7 & $3139^{a}$ \\
\hline & & Pooled SEM & 0.33 & 0.15 & 0.19 & 0.26 & 0.26 & 8.23 \\
\hline & & RGM & & & & & & \\
\hline & & 0 & $74.1^{b}$ & $56.2^{b}$ & $77.1^{b}$ & 33.6 & 39.3 & $3205^{b}$ \\
\hline & & 15 & $74.1^{b}$ & $56.5^{\mathrm{b}}$ & $77.2^{\mathrm{b}}$ & 33.9 & 39.8 & $3205^{\mathrm{b}}$ \\
\hline & & 17.5 & $71.4^{\mathrm{a}}$ & $55.4^{\mathrm{a}}$ & $76.2^{\mathrm{a}}$ & 33.5 & 39.3 & $3167^{\mathrm{a}}$ \\
\hline & & Enzyme & & & & & & \\
\hline & & - & $72.1^{\mathrm{a}}$ & $55.6^{\mathrm{a}}$ & $76.2^{\mathrm{a}}$ & 33.1 & 38.8 & $3162^{a}$ \\
\hline & & $X$ & $73.8^{b}$ & $56.5^{\mathrm{a}}$ & $78.1^{\mathrm{c}}$ & 33.7 & 40.1 & $3238^{b}$ \\
\hline & & $\mathrm{P}$ & $75.4^{\mathrm{c}}$ & $57.2^{\mathrm{b}}$ & $77.1^{\mathrm{b}}$ & 33.9 & 39.4 & $3214^{\mathrm{b}}$ \\
\hline & & $\mathrm{M}$ & $71.6^{\mathrm{a}}$ & $56.0^{\mathrm{a}}$ & $76.0^{\mathrm{a}}$ & 33.8 & 39.5 & $3155^{\mathrm{a}}$ \\
\hline & & Significance & & & & & & \\
\hline & & RGM & $\mathrm{P}<0.01$ & $\mathrm{P}<0.01$ & $\mathrm{P}<0.05$ & NS & NS & $\mathrm{P}<0.01$ \\
\hline & & Enzyme & $\mathrm{P}<0.01$ & $\mathrm{P}<0.05$ & $\mathrm{P}<0.01$ & NS & NS & $\mathrm{P}<0.01$ \\
\hline & & Interaction & $\mathrm{P}<0.01$ & $\mathrm{P}<0.01$ & NS & NS & NS & $\mathrm{P}<0.01$ \\
\hline
\end{tabular}

Values bearing different superscripts within the column differ significantly* $(\mathrm{P}<0.01), * *(\mathrm{P}<0.05)$ and NS: Non-significant $(\mathrm{P}>0.05)$.

and protease enzymes as compared to without enzyme and multienzymes supplemented groups.

Interaction of RGM and enzymes showed no significant $(\mathrm{P}>0.05)$ difference in nutrient utilization between different dietary treatments and control in gross energy metabolizability (GEM), calcium and phosphorous retention (\%). Dry matter metabolizability (DMM) was significantly $(\mathrm{P}<0.01)$ lower in $\mathrm{T} 8, \mathrm{~T} 9, \mathrm{~T} 10$ and T12 groups as compared to control and other dietary treatments. However, dietary treatments T2, T3, T6, T7 and $\mathrm{T} 11$ were significantly $(\mathrm{P}<0.01)$ better in $\mathrm{DMM}$ as compared to control and other dietary treatments. Nitrogen retention (\%) was significantly $(\mathrm{P}<0.05)$ lower in $17.5 \%$ RGM without enzyme group as compared to control and other dietary treatments, but other dietary treatments did not differ significantly $(\mathrm{P}>0.05)$ from control. Apparent metabolizable energy (AME) of the diets were significantly
$(\mathrm{P}<0.01)$ higher in dietary treatments $\mathrm{T} 2, \mathrm{~T} 4, \mathrm{~T} 6, \mathrm{~T} 7$ and $\mathrm{T} 10$ as compared to control and other dietary treatments.

Protein efficiency ratio (PER) as affected by feeding different levels of RGM with or without enzymes are tabulated in Table 7. There was no significant $(\mathrm{P}>0.05)$ difference in pre-starter, starter, finisher and overall phases by incorporating different levels of RGM $(0,15$ and $17.5 \%)$ in PER. The PER was significantly $(\mathrm{P}<0.01)$ better in protease and multienzymes groups as compared to without enzyme groups, but it did not show any significant $(\mathrm{P}>0.05)$ difference from xylanase enzyme groups during pre-starter and overall phases. Enzyme (X,P and M) supplementation on PER revealed that no significant $(\mathrm{P}>0.05)$ difference was found during starter phase. However in finisher phase, enzyme supplemented $(\mathrm{X}, \mathrm{P}$ and $\mathrm{M})$ groups were significantly $(\mathrm{P}<0.01)$ better than without enzyme supplementation in terms of PER. 
Table 7: Effect of feeding different level of RGM with or without enzymes on protein efficiency ratio (PER)

\begin{tabular}{|c|c|c|c|c|c|c|}
\hline Treatment & RGM \% & Enzyme & Prestarter & Starter & Finisher & Overall \\
\hline T1 & 0 & - & $4.19^{a}$ & 3.46 & 2.56 & $2.91^{\mathrm{ab}}$ \\
\hline $\mathrm{T} 2$ & 0 & $\mathrm{X}$ & $4.27^{\mathrm{ab}}$ & 3.27 & 2.68 & $2.97^{\mathrm{abc}}$ \\
\hline $\mathrm{T} 3$ & 0 & $\mathrm{P}$ & $4.22^{\mathrm{ab}}$ & 3.33 & 2.66 & $2.96^{\mathrm{abc}}$ \\
\hline $\mathrm{T} 4$ & 0 & M & $4.71^{c}$ & 3.54 & 2.81 & $3.16^{\mathrm{d}}$ \\
\hline $\mathrm{T} 5$ & 15 & - & $4.16^{\mathrm{a}}$ & 3.42 & 2.63 & $2.95^{\mathrm{abc}}$ \\
\hline T6 & 15 & $\mathrm{X}$ & $4.29^{\mathrm{ab}}$ & 3.44 & 2.63 & $2.96^{\mathrm{abc}}$ \\
\hline $\mathrm{T} 7$ & 15 & $\mathrm{P}$ & $4.60^{c}$ & 3.34 & 2.75 & $3.06^{\mathrm{cd}}$ \\
\hline T8 & 15 & M & $4.21^{\mathrm{ab}}$ & 3.36 & 2.69 & $2.99^{\mathrm{abc}}$ \\
\hline T9 & 17.5 & - & $4.15^{\mathrm{a}}$ & 3.32 & 2.54 & $2.87^{\mathrm{a}}$ \\
\hline $\mathrm{T} 10$ & 17.5 & $X$ & $4.26^{\mathrm{ab}}$ & 3.21 & 2.72 & $2.98^{\mathrm{abc}}$ \\
\hline T11 & 17.5 & $\mathrm{P}$ & $4.51^{b c}$ & 3.35 & 2.70 & $3.02^{b c}$ \\
\hline \multirow[t]{15}{*}{$\mathrm{T} 12$} & 17.5 & M & $4.24^{\mathrm{ab}}$ & 3.38 & 2.66 & $2.96^{\mathrm{abc}}$ \\
\hline & & Pooled SEM & 0.03 & 0.02 & 0.02 & 0.02 \\
\hline & & RGM & & & & \\
\hline & & 0 & 4.35 & 3.40 & 2.68 & 3.00 \\
\hline & & 15 & 4.32 & 3.39 & 2.67 & 2.99 \\
\hline & & 17.5 & 4.29 & 3.31 & 2.65 & 2.96 \\
\hline & & Enzyme & & & & \\
\hline & & - & $4.17^{\mathrm{a}}$ & 3.40 & $2.57^{\mathrm{a}}$ & $2.91^{\mathrm{a}}$ \\
\hline & & $\mathrm{X}$ & $4.27^{\mathrm{ab}}$ & 3.30 & $2.67^{b}$ & $2.97^{\mathrm{ab}}$ \\
\hline & & $\mathrm{P}$ & $4.44^{\mathrm{c}}$ & 3.34 & $2.70^{\mathrm{b}}$ & $3.02^{\mathrm{b}}$ \\
\hline & & M & $4.38^{\mathrm{bc}}$ & 3.43 & $2.72^{b}$ & $3.04^{b}$ \\
\hline & & Significance & & & & \\
\hline & & RGM & NS & $\mathrm{NS}$ & NS & NS \\
\hline & & Enzyme & $\mathrm{P}<0.01$ & NS & $\mathrm{P}<0.01$ & $\mathrm{P}<0.01$ \\
\hline & & Interaction & $\mathrm{P}<0.01$ & NS & NS & $\mathrm{P}<0.05$ \\
\hline
\end{tabular}

Values bearing different superscripts within the column differ significantly* $(\mathrm{P}<0.01), * *(\mathrm{P}<0.05)$ and NS:Non-significant $(\mathrm{P}>0.05)$.

Table 8: Effect of feeding different level of RGM with or without enzymes on energy efficiency ratio (EER)

\begin{tabular}{|c|c|c|c|c|c|c|}
\hline Treatment & RGM \% & Enzyme & Prestarter & Starter & Finisher & Overall \\
\hline $\mathrm{T} 1$ & 0 & - & $30.36^{\mathrm{a}}$ & 24.01 & 15.80 & $18.63^{\mathrm{ab}}$ \\
\hline $\mathrm{T} 2$ & 0 & $\mathrm{X}$ & $30.92^{\mathrm{ab}}$ & 22.72 & 16.53 & $19.04^{\mathrm{abc}}$ \\
\hline $\mathrm{T} 3$ & 0 & $\mathrm{P}$ & $30.58^{\mathrm{ab}}$ & 23.16 & 16.38 & $18.99^{\mathrm{abc}}$ \\
\hline $\mathrm{T} 4$ & 0 & M & $34.14^{\mathrm{c}}$ & 24.64 & 17.35 & $20.21^{\mathrm{d}}$ \\
\hline $\mathrm{T} 5$ & 15 & - & $30.14^{\mathrm{a}}$ & 23.76 & 16.21 & $18.90^{\mathrm{abc}}$ \\
\hline T6 & 15 & X & $33.34^{\mathrm{c}}$ & 23.87 & 16.18 & $18.99^{\mathrm{abc}}$ \\
\hline $\mathrm{T} 7$ & 15 & $\mathrm{P}$ & $31.07^{\mathrm{ab}}$ & 23.16 & 16.93 & $19.63^{\mathrm{cd}}$ \\
\hline $\mathrm{T} 8$ & 15 & M & $30.46^{\mathrm{ab}}$ & 23.36 & 16.61 & $19.16^{\mathrm{abc}}$ \\
\hline T9 & 17.5 & - & $30.08^{a}$ & 23.06 & 15.64 & $18.39^{\mathrm{a}}$ \\
\hline $\mathrm{T} 10$ & 17.5 & $\mathrm{X}$ & $32.68^{\mathrm{bc}}$ & 22.28 & 16.76 & $19.14^{\mathrm{abc}}$ \\
\hline $\mathrm{T} 11$ & 17.5 & $\mathrm{P}$ & $30.85^{\mathrm{ab}}$ & 23.28 & 16.60 & $19.37^{b c}$ \\
\hline $\mathrm{T} 12$ & 17.5 & M & $30.71^{\mathrm{ab}}$ & 23.45 & 16.37 & $19.01^{\mathrm{abc}}$ \\
\hline
\end{tabular}




\begin{tabular}{llllll} 
& Pooled SEM & 0.25 & 0.16 & 0.10 & 0.09 \\
\cline { 2 - 6 } RGM & 0 & 31.50 & 23.63 & 16.52 & 19.22 \\
& 15 & 31.26 & 23.54 & 16.48 & 19.17 \\
& 17.5 & 31.28 & 23.39 & 16.45 & 19.13 \\
\hline Enzyme & & & & $18.64^{\mathrm{a}}$ \\
& - & $30.20^{\mathrm{a}}$ & 23.61 & $15.88^{\mathrm{a}}$ & $19.06^{\mathrm{b}}$ \\
& $\mathrm{X}$ & $32.20^{\mathrm{c}}$ & 22.95 & $16.48^{\mathrm{b}}$ & $19.33^{\mathrm{b}}$ \\
& $\mathrm{P}$ & $30.95^{\mathrm{ab}}$ & 23.20 & $16.63^{\mathrm{b}}$ & $19.46^{\mathrm{b}}$ \\
\hline $\mathrm{M}$ & $31.77^{\mathrm{bc}}$ & 23.82 & $16.77^{\mathrm{b}}$ & $\mathrm{NS}$ \\
& Significance & & & $\mathrm{P}<0.01$ \\
\hline RGM & $\mathrm{NS}$ & $\mathrm{NS}$ & $\mathrm{NS}$ & $\mathrm{P}<0.05$ \\
\hline
\end{tabular}

Values bearing different superscripts within the column differ significantly $*(\mathrm{P}<0.01), * *(\mathrm{P}<0.05)$ and NS-Non-significant $(\mathrm{P}>0.05)$.

Interaction of RGM and enzymes showed that $0 \%$ RGM with multienzymes and 15\%RGM with protease were significantly $(\mathrm{P}<0.01)$ better than control and other dietary treatments, but they did not show any significant $(\mathrm{P}>0.05)$ difference from $17.5 \%$ RGM with protease during prestarter phase. No significant $(\mathrm{P}>0.05)$ difference was found in PER between different dietary treatments and control during starter and finisher phases. However in overall phase, $0 \%$ RGM with multienzymes were significantly $(\mathrm{P}<0.05)$ better as compared to control, but it did not differ significantly ( $\mathrm{P}>0.05)$ from $15 \%$ RGM with protease group.

Energy efficiency ratio (EER) as affected by feeding different levels of RGM with or without enzymes are tabulated in Table 8. There was no significant $(\mathrm{P}>0.05)$ difference in pre-starter, starter, finisher and overall phases by incorporating different levels of RGM $(0,15$ and $17.5 \%)$ in EER. Enzymes supplementation (P, $\mathrm{X}$ and $\mathrm{M})$ on EER revealed that during pre-starter phase, xylanase enzyme supplementation was significantly $(\mathrm{P}<0.01)$ better as compared to protease and without enzyme supplementation, but it did not show any significant $(\mathrm{P}>0.05)$ difference from multienzymes supplementation. There was no significant $(\mathrm{P}>0.05)$ difference during starter phase in EER. However in finisher and overall phases, enzyme (X, P and $M$ ) supplementation were significantly $(\mathrm{P}<0.01)$ better than without enzyme supplementation in EER. Interaction of RGM and enzymes showed that during pre-starter phase, $0 \%$ RGM with multienzymes and $15 \%$ RGM with xylanase significantly $(\mathrm{P}<0.01)$ better than control and other dietary treatments, but they did not show any significant $(\mathrm{P}>0.05)$ difference from $17.5 \%$ RGM with xylanase in EER. No significant $(\mathrm{P}>0.05)$ difference was found in EER between different dietary treatments and control during starter and finisher phases. However in overall phase, 0\% RGM with multienzymes was significantly $(\mathrm{P}<0.05)$ better than control and other dietary treatments, but it did not show any significant $(\mathrm{P}>0.05)$ difference from $15 \% \mathrm{RGM}$ with protease.

Our results are in agreement with Metwally and Farhat (2015) and Wani (2017) who reported no significant difference in nutrient digestibility (DM, CP, EE, NDF and starch) up to addition of $12.5 \%$ and $20 \%$ RGM in diet of broiler chicken respectively. Our results are in disagreement with Kumar et al. (2016), who reported no significant $(\mathrm{P}>0.05)$ difference in digestibility of nutrients and nitrogen balance on addition of RGM up to $21 \%$ in the diet of growing dairy calves. Effect of enzyme supplementation found beneficial in our study in terms of nutrient utilization. Contrary to our finding, Wani (2017) reported protease enzyme supplementation did not reveal any significant $(\mathrm{P}>0.05)$ difference on nutrient utilization of broiler chicken up to $20 \%$ inclusion level of RGM. Poor nutrient utilization of RGM may be associated with level and type of crude fiber in RGM along with poor digestibility of RGM as compared to soybean meal. Better nutrient utilization in our experiment may be due to improvement in RGM digestibility by different enzymes supplementation. 


\section{CONCLUSION}

Thus, it may be concluded that protease enzyme supplementation was found best in RGM diet and multienzymes supplementation was found best in cornsoya diet to improve their feeding value. Protease enzyme supplementation can increase the effective and safe inclusion level of RGM from 15 to $17.5 \%$ for economic broiler production.

\section{ACKNOWLEDGEMENTS}

ICAR-Central avian research institute, Izatnagar, Utter Pradesh-243122 for providing all necessary inputs and facilities.

\section{REFERENCES}

AOAC, 2000. Association of official analytical chemists. Official methods of analysis. $17^{\text {th }}$ edn., Washington, DC.

Agricultural Statistics. 2018. Agricultural statistics at a glance. Department of agriculture, cooperation and farmers welfare. Government of India, New Delhi.

Chessson, A. 2001. Non starch polysaccharide degrading enzymes in poultry diets: influence of ingredients on the selection of activities. World Poult. Sci., 57: 251-262.

Giannenas, I., Bonos, E., Anestis, V., Filioussis, G., Papanastasiou, D.K., Bartzanas, T., apaioannou, N., Tzora, A. and Skoufos, I. 2017. Effects of protease addition and replacement of soybean meal by corn gluten meal on the growth of broilers and on the environmental performances of a broiler production system in greece. PloS One, 12(1): e0169511.
Gopalkrishnan, M.V. and Jamuna, P. 2000. Optimum time requirement for enzymatic hydrolysis of food proteins. $J$. Food Sci. Technol., 37: 319-322.

ICAR.2013. Nutrient requirements of Animals-Poultry. Indian Council of Agriculture Research, New Delhi, India. 1:13-16.

Kamra, D.N. and Agarwal, N. 2003.Techniques in rumen microbiology. I.V.R.I.D.U, Izatnagar,U.P. India.

Kumar, R., Thakur, S.S. and Mahesh, M.S. 2016. Rice gluten meal as an alternative by product feed for growing dairy calves. Trop. Anim. Health Prod., 48(3): 619-624.

Malik, T.A., Thakur, S.S., Mahesh, M.S. and Yogi, R.K. 2017. Replacing groundnut cake with gluten meals of rice and maize in diets for growing Sahiwal cattle. Asia-Australas $J$. Anim. Sci., 30(10): 1410.

Metwally, A. and Farahat, M. 2015. Nutritive value and feeding of rice gluten meal in broiler chickens. Res. Opin. Anim. Vet. Sci., 5: 443-451.

Sherazi, T.H., Alam, M.Z., GiIani, A.H. and Nawaz, H. 1995. Graded replacement of fish meal with rice protein meal in broiler ration. Pak. J. Agric. Sci., 32: 2-3.

Snedecor, G.W. and Cochran, W.G. 1989. Statistical Methods, $7^{\text {th }}$ edn. Oxford and IBH. Iowa State University Press, Iowa, USA.

Tukey, J. 1949. Comparing individual means in the analysis of variance. Biometrics, 5(2): 99-114.

Wani, M.A. 2017. Evaluation of feeding value of rice gluten meal in broiler chicken. Ph.D. thesis submitted to IVRIDU, Izatnagar, U.P., India.

Wani, M.A., Tyagi, P.K., Mir, N.A., Hazarika R., Sheikh, S.A., Tyagi, P.K., Dinani, O.P., Mandal, A.B. 2018. Feeding value of rice gluten meal as an alternate protein source in broiler chickens. Turk. J. Vet. Anim. Sci., 42: 473-479. 
